\title{
Statin treatment increases lipoprotein(a) levels in subjects with low molecular weight apolipoprotein(a) phenotype
}

\author{
Reyhana Yahya ${ }^{\mathrm{a}}$, Kirsten Berk ${ }^{\mathrm{a}, \mathrm{b}}$, Adrie Verhoeven ${ }^{\mathrm{a}}$, Sven Bos ${ }^{\mathrm{a}}$, Leonie van der Zee ${ }^{\mathrm{a}}$, \\ Jeanette Touw ${ }^{\mathrm{a}}$, Gertraud Erhart ${ }^{\mathrm{d}}$, Florian Kronenberg ${ }^{\mathrm{d}}$, Reinier Timman ${ }^{\mathrm{c}}$, Eric Sijbrands ${ }^{\mathrm{a}}$, \\ Jeanine Roeters van Lennep ${ }^{\mathrm{a}}$, Monique Mulder ${ }^{\mathrm{a}, "}$ \\ ${ }^{a}$ Department of Internal Medicine, division of Vascular Medicine and Pharmacology, Division of Medical Psychology and Psychotherapy, Erasmus MC, Erasmus University \\ Medical Center, Rotterdam, Netherlands \\ ${ }^{\mathrm{b}}$ Department of Dietetics and Division of Medical Psychology and Psychotherapy, Erasmus MC, Erasmus University Medical Center, Rotterdam, Netherlands \\ ${ }^{\mathrm{c}}$ Department of Psychiatry, Division of Medical Psychology and Psychotherapy, Erasmus MC, Erasmus University Medical Center, Rotterdam, Netherlands \\ ${ }^{\mathrm{d}}$ Division of Genetic Epidemiology, Department of Medical Genetics, Molecular and Clinical Pharmacology, Medical University of Innsbruck, Innsbruck, Austria
}

\section{H I G H L I G H T S}

- High levels of Lp(a) may contribute to the residual cardiovascular risk in statin-treated dyslipidemic patients.

- Reported data on modulatory effects of statins on plasma Lp(a) levels are inconsistent.

- Statin treatment increases Lp(a) levels in patients with dyslipidemia, exclusively in those with the LMW apo(a) phenotype.

- The increase in Lp(a) levels upon statin treatment was not associated with common LPA SNPs or change in LDL cholesterol.

\section{A R T I C L E I N F O}

\section{Keywords:}

Statin

Dyslipidemia

lipoprotein(a)

KIV2

LPA gene

\begin{abstract}
A B S T R A C T
Background and aims: We aimed to evaluate the effect of statin treatment initiation on lipoprotein(a) [Lp(a)] levels in patients with dyslipidemia, and the interactions with the apolipoprotein(a) [apo(a)] phenotype, $L P A$ single nucleotide polymorphisms (SNPs) and change in LDL cholesterol.

Methods: The study population consisted of patients with dyslipidemia, predominantly familial hypercholesterolemia, who first initiated statin treatment (initiation group; $n=39$ ) or were already on stable statin treatment for at least 4 months (control group; $n=42$ ). Plasma Lp(a) levels were determined with a particle-enhanced immunoturbidimetric assay before and at least 2 months after start of statin treatment in individuals of the initiation group, and at two time points with an interval of at least 2 months in the control group. High and low molecular weight (HMW and LMW, respectively) apo(a) phenotype was determined by immunoblotting, and the common LPA SNPs rs10455872, rs3798220 and rs41272110 by Taqman assay.

Results: Plasma Lp(a) levels did not increase significantly in the initiation group (median 20.5 (IQR 10.9-80.7) to $23.3(10.8-71.8) \mathrm{mg} / \mathrm{dL} ; p=0.09$ ) nor in the control group (30.9 (IQR 9.2-147.0) to 31.7 (IQR 10.9-164.0) $\mathrm{mg} / \mathrm{dL} ; p=0.61$ ). In patients with the LMW apo(a) phenotype, Lp(a) levels increased significantly from 66.4 (IQR 23.5-148.3) to 97.4 (IQR 24.9-160.4) $\mathrm{mg} / \mathrm{dL}(p=0.026$ ) in the initiation group, but not in the control group and not in patients characterized by the HMW apo(a) phenotype. Interactions with common LPA SNPs and change in LDL cholesterol were not significant.

Conclusions: Statins affect Lp(a) levels differently in patients with dyslipidemia depending on the apo(a) phenotype. Statins increase Lp(a) levels exclusively in patients with the LMW apo(a) phenotype.
\end{abstract}

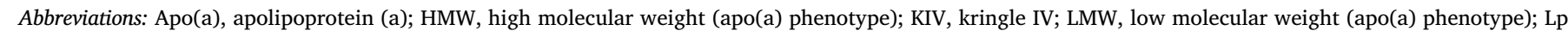
(a), Lipoprotein (a)

* Corresponding author. Department of Internal Medicine, Erasmus MC, Erasmus University Medical Center, Wytemaweg 80, 3015 CN, Rotterdam, the Netherlands

E-mail address: m.t.mulder@erasmusmc.nl (M. Mulder). 


\section{Introduction}

An elevated plasma lipoprotein(a) [Lp(a)] level is an established independent risk factor for cardiovascular disease (CVD) [1-4]. High levels of Lp(a) may contribute to the considerable residual cardiovascular risk in statin-treated dyslipidemic patients including those with familial hypercholesterolemia (FH) with LDL-cholesterol below target levels [5,6]. Lp(a) is an LDL-like particle with an apolipoprotein(a) [apo (a)] covalently attached to apolipoprotein B100. Lp(a) levels are highly genetically determined by the number of kringle IV (KIV) copies in the LPA gene encoding apo(a) [7-12]. Five-to ten-fold higher plasma Lp(a) levels are found in individuals with at least one low molecular weight (LMW) apo(a) ( $\leq 22 \mathrm{KIV}$ repeats) than in individuals only with high molecular weight (HMW) apo(a) ( $\geq 23 \mathrm{KIV}$ repeats) [13]. Several single nucleotide polymorphisms (SNPs) in the LPA gene are strongly associated with Lp(a) levels and CVD [11,12,14,15]. Although to a limited extent, non-genetic factors can affect Lp(a) plasma levels, such as for example weight reduction [16], high saturated fat intake [17,18] or kidney impairment [19]. Whether statins modify Lp(a) levels is not clear as inconsistent data have been reported [20-22]. Moreover, the underlying mechanisms remain to be clarified [21-23]. We hypothesized that the effect of statin treatment on Lp(a) levels depends on the KIV copy number and/or sequence variations in the LPA gene [24].

To investigate the effect of statin treatment on plasma $L p(a)$ levels we determined changes in Lp(a) levels over time in dyslipidemic patients during the first few months after initiation of statin therapy. As a control we measured plasma Lp(a) levels at two distinct time points in dyslipidemic patients who were on stable statin treatment for at least 4 months. Secondly, we examined whether changes in plasma Lp(a) level induced by statin treatment were associated with apo(a) phenotype, common SNPs in the LPA gene and change in LDL cholesterol level.

\section{Materials and methods}

\subsection{Study population}

Patients aged $\geq 18$ years were consecutively (2011-2015) recruited at the outpatient cardiovascular genetics clinic at the Erasmus MC, Rotterdam, the Netherlands. The two study groups both consisted of patients with dyslipidemia, mostly $(\sim 85 \%)$ familial hypercholesterolemia ( $\mathrm{FH})$, and were primarily of Caucasian origin. In the initiation group, patients started statin treatment for the first time $(n=39)$, whereas in the control group patients were on stable statin treatment for at least 4 months $(n=42)$. Patients from the initiation group were newly referred to our tertiary center whereas patients from the control group were already seen at the outpatient clinic for follow up.

Fasting blood samples were obtained from each patient at 2 different time points with an interval of at least 2 months. In the initiation group the first time point was immediately before the initiation of statin treatment. Patients included in the control group did not change statin type or dose or use of other lipid-lowering drugs between the two blood sampling time points.

All participants provided written informed consent for blood collection and analysis, sample storage, and usage of biometrical data. This study was approved by the medical ethical committee of the Erasmus MC.

\subsection{Anthropometric and laboratory measurements}

Patient characteristics were recorded at baseline. Whole blood and plasma samples were stored at $-80{ }^{\circ} \mathrm{C}$ until further analysis. Total cholesterol, LDL- and HDL-cholesterol (LDL-C, HDL-C), triglycerides (TG) and apoB100 were measured using standard laboratory techniques. 'True LDL-C' was determined by subtracting Lp(a)-cholesterol from LDL-C. Lp(a)-cholesterol was calculated from the Lp(a) levels in $\mathrm{nmol} / \mathrm{L}$ with the assumptions that the molecular cholesterol-to-
apoB100 ratio, and hence the cholesterol-to-apo(a) ratio, is similar to that of LDL, and that LDL consists of $25 \%$ apoB100 protein en $50 \%$ cholesterol by weight, as described by Viney et al. [25].

Plasma Lp(a) levels ( $\mathrm{mg} / \mathrm{dL}$ or $\mathrm{nmol} / \mathrm{L})$ were measured with a particle-enhanced immunoturbidimetric assay, which is largely independent of apo(a) kringle IV (KIV) type 2 copy number (Diagnostic System \#171399910930; DiaSys Diagnostic System, GmbH, Germany) [26]. Baseline and follow-up samples of all participants were measured in the same run and both samples were close to each other on the plate. The detection limit of the assay was $3.0 \mathrm{mg} / \mathrm{dL}$ and the mean intraassay variability was $2.8 \%$. Sampling at two different time points in 28 healthy controls with an interval of 2-4 months did not reveal significant changes in Lp(a) level [27]. At baseline, the number of apo(a) KIV repeats was determined by SDS agarose gel electrophoresis and immunoblotting, as previously described [26]. When two apo(a) isoforms were identified in the blot, either the number of KIV repeats of the smallest isoform or the mean repeat number of both isoforms was used in the analysis. According to the number of KIV repeats, patients were classified as having an LMW apo(a) phenotype when they expressed at least one apo(a) isoform with $\leq 22 \mathrm{KIV}$ repeats, or HMW apo (a) phenotype when they expressed only apo(a) isoforms with $\geq 23 \mathrm{KIV}$ repeats.

DNA was isolated using the Invisorb ${ }^{\bullet}$ Blood Universal Kit from Stratec Molecular (Berlin, Germany). Common variants of the LPA gene (rs10455872, rs3798220 and rs41272110 (T3888P)) [15,28] were genotyped with TaqMan allelic discrimination assays, designed and optimized by Applied Biosystems (Foster City, CA, USA) on a Taqman Prism 7900 HT platform.

\subsection{Statistical analysis}

Normality of the data was tested with Shapiro-Wilks test. Differences between the intervention and the control group at baseline were tested using $\chi^{2}$ tests with continuity correction, independent samples $t$ tests or Mann-Whitney $U$ tests, according to the distribution of the data. The difference in Lp(a) level within the groups was analysed using Wilcoxon signed ranks tests. The relationship between change in Lp(a) level over time and apo(a) KIV repeat number was analysed with Spearman's rank order correlation test. Results with $p$ values of $<0.05$ were considered statistically significant. SPSS version 24.0 (IBM corp., Armonk, New York, USA) and GraphPad Prism version 5 (GraphPad Software, La Jolla, California, USA) were used for the statistical analyses.

\section{Results}

\subsection{Patient characteristics}

Table 1 shows the patient characteristics at baseline. The initiation group was younger and had higher baseline levels of total cholesterol, LDL-C and apoB100 than the control group. The median interval between the two blood sampling time points was 3.8 months (range 2-20 months) in the initiation group and 11.7 months (range 4-26 months) in the control group.

\subsection{Lipoprotein (a) levels}

At baseline, median Lp(a) levels were 20.5 (IQR 10.9-80.7) mg/dL for the initiation group and 30.9 (IQR 9.2-147.0) $\mathrm{mg} / \mathrm{dL}$ for the control group (Mann-Whitney $\mathrm{U}(\mathrm{MWU})=702.5 ; \mathrm{Z}=-1.101 ; p=0.27$ ). At the second time point, the median Lp(a) level of the initiation group was 23.3 (IQR 10.8-71.8) $\mathrm{mg} / \mathrm{dL}$, and 31.7 (IQR 10.9-164.0) $\mathrm{mg} / \mathrm{dL}$ for the control group (MWU $=705.0 ; \mathrm{Z}=-1.078 ; \mathrm{p}=0.28$ ), which corresponds to a relative change of $13.7 \%$ (Wilcoxon $Z=1.697 ; p=0.09$ ) and $2.6 \%(Z=0.518 ; p=0.61)$, respectively. We found no difference between the two groups in the change of $\mathrm{Lp}$ (a) levels (MWU = 729.0; $\mathrm{Z}=-0.851 ; p=0.40)$. 
Table 1

Baseline characteristics.

\begin{tabular}{|c|c|c|c|}
\hline Characteristics & Initiation group $n=39$ & Control group $n=42$ & $p$ \\
\hline Age (years) & $39.9 \pm 16.3$ & $52.9 \pm 14.2$ & $<0.001$ \\
\hline Sex (male) & $18(46 \%)$ & $22(52 \%)$ & 0.74 \\
\hline Follow-up time (months) & $3.8[3.1,5.7]$ & $11.7[11.0,23.1]$ & $<0.001$ \\
\hline $\mathrm{FH}^{\mathrm{a}}$ & $33(85 \%)$ & $37(88 \%)$ & 0.90 \\
\hline LMW apo(a) phenotype & $14(36 \%)$ & $14(33 \%)$ & 0.99 \\
\hline rs10455872 & $4(11 \%)$ & $8(19 \%)$ & 0.45 \\
\hline rs $3798220^{\mathrm{b}}$ & $0(0 \%)$ & $4(10 \%)$ & 0.17 \\
\hline rs $41272110^{\mathrm{b}}$ & $15(40 \%)$ & $11(26 \%)$ & 0.30 \\
\hline Body mass index $\left(\mathrm{kg} / \mathrm{m}^{2}\right)$ & $25.2 \pm 3.5$ & $28.1 \pm 5.8$ & 0.008 \\
\hline Systolic blood pressure $(\mathrm{mmHg})$ & $128 \pm 19$ & $135 \pm 11$ & 0.05 \\
\hline Diastolic blood pressure $(\mathrm{mmHg})$ & $78 \pm 9$ & $81 \pm 8$ & 0.23 \\
\hline Hypertension ${ }^{c}$ & $9(23 \%)$ & $16(38 \%)$ & 0.22 \\
\hline Diabetes mellitus ${ }^{\mathrm{c}}$ & $1(3 \%)$ & $4(10 \%)$ & 0.40 \\
\hline Smoker $^{\mathrm{c}}$ (current, former) & $8(21 \%)$ & $7(17 \%)$ & 0.66 \\
\hline Premature CVD patient ${ }^{\mathrm{c}}$ & $3(8 \%)$ & $9(21 \%)$ & 0.15 \\
\hline Total Cholesterol $(\mathrm{mmol} / \mathrm{L})$ & $7.5 \pm 1.9$ & $4.5 \pm 0.6$ & $<0.001$ \\
\hline LDL-C (mmol/L) & $5.5 \pm 1.6$ & $2.6 \pm 0.6$ & $<0.001$ \\
\hline 'True LDL-C' $(\mathrm{mmol} / \mathrm{L})^{\mathrm{d}}$ & $5.3 \pm 1.6$ & $2.1 \pm 0.9$ & $<0.001$ \\
\hline HDL-C (mmol/L) & $1.3 \pm 0.4$ & $1.4 \pm 0.5$ & 0.67 \\
\hline Triglyceride (mmol/L) & $1.8 \pm 2.0$ & $1.9 \pm 1.9$ & 0.92 \\
\hline Apolipoprotein B $100(\mu \mathrm{mol} / \mathrm{L})$ & $1.5 \pm 0.3$ & $0.9 \pm 0.2$ & $<0.001$ \\
\hline Lipoprotein(a) (mg/dL) & 20.5 [10.9-80.7] & $30.9[9.2-147.0]$ & 0.27 \\
\hline Apo(a) KIV repeat number ${ }^{\mathrm{e}}$ & $27.1 \pm 5.6$ & $28.0 \pm 6.3$ & 0.65 \\
\hline LMW apo(a) phenotype & $14(36 \%)$ & $14(33 \%)$ & 0.81 \\
\hline
\end{tabular}

Data are mean \pm SD or median [IQR]

a Percentage subjects with familial hypercholesterolemia (FH) either genetically or clinically confirmed [39].

b Carriers of the minor allele.

c Percentage subjects smoking (current and former), hypertension (defined as a systolic blood pressure $>140 \mathrm{mmHg}$ or a diastolic blood pressure $>90 \mathrm{mmHg}$ or the use of anti-hypertensive medication), diabetes mellitus (defined as a fasting glucose $>7 \mathrm{mmol} / \mathrm{L}$ or the use of antidiabetic medication), and premature CVD (defined as a myocardial infarction, stroke or peripheral artery disease with intervention before age 55 for men and 60 years for women)

d LDL-C after correction for $\operatorname{Lp}(a)$-cholesterol.

e The KIV repeat number of the smallest apo(a) isoform when two different apo(a) isoforms are present on the immunoblot.

\subsection{Association with apo(a) phenotypes}

In both the initiation and control group, 14 patients (36\% and 33\%, respectively) had LMW apo(a) phenotype $\left(\chi_{(1)}^{2}=0.000 ; p=0.99\right)$. As expected, Lp(a) levels at baseline were higher in patients with the LMW phenotype than with the HMW phenotype (137.8 (IQR 36.8-206.5) vs 15.3 (IQR 6.8-31.5) $\mathrm{mg} / \mathrm{dL}$; $\mathrm{MWU}=184.5, \mathrm{Z}=-5.537 ; p<0.001$; control group and initiation group combined). Fig. 1 shows the change in Lp(a) by group and apo(a) phenotype. Upon starting statin therapy Lp(a) increased from 66.4 (IQR 23.5-148.3) to 97.4 (IQR 24.9-160.4) $\mathrm{mg} / \mathrm{dL}$ ( $\mathrm{Z}=2.229 ; p=0.026)$ in patients with the LMW apo(a) phenotype, but not in patients with the HMW apo(a) phenotype (14.0 (IQR 8.1-36.8) to 15.1 (IQR 9.5-34.5) $\mathrm{mg} / \mathrm{dL}(\mathrm{Z}=0.152 ; p=0.879)$ ). The change in Lp(a) upon starting statin therapy was different between the LMW and HMW subgroups (MWU $=91.5 ; \mathrm{Z}=-2.445 ; p=0.013$ ). In the control group, Lp(a) levels did not change over time in the LMW subgroup (180.7 (IQR 109.7-236.3) to 190.2 (IQR 97.4-233.3) $\mathrm{mg} / \mathrm{dL} ; \mathrm{Z}=0.596 ; p=0.55$ ), nor in the HMW subgroup (15.6 (IQR 5.9-31.0) to 16.9 (IQR 7.2-34.1) $\mathrm{mg} / \mathrm{dL} ; \mathrm{Z}=1.601 ; p=0.11$ ). Qualitatively similar results were obtained when $\mathrm{Lp}(\mathrm{a})$ levels were expressed in nmol/L.

Next, we analyzed the relationship between Lp(a) levels and the number of KIV repeats of the smallest apo(a) isoform in the case two apo(a) isoforms were expressed. Baseline Lp(a) levels were strongly correlated with the apo(a) KIV repeat number in both the initiation and the control group $(\mathrm{r}=-0.589 ; p<0.001$ and $\mathrm{r}=-0.802 ; p<0.001$, respectively). The change in $\mathrm{Lp}(\mathrm{a})$ level over time significantly correlated with the KIV repeat number in the initiation group but not in the control group (Spearman's rho $-0.424 ; p=0.007$ and $0.079 ; p=0.62$, respectively). The change in $\mathrm{Lp}(\mathrm{a})$ level remained negatively correlated with apo(a) KIV repeat numbers after correction for baseline Lp(a)

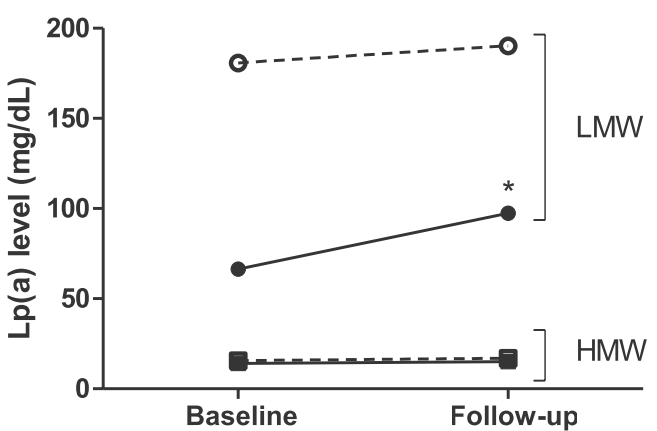

Fig. 1. Lp(a) levels at baseline and at follow-up in the subgroups.

This figure shows baseline and follow-up Lp(a) levels in patients with the LMW and HMW apo(a) phenotype. The continuous line indicates the initiation group and the dotted line indicates the control group. ${ }^{*} p<0.05$.

(after Blom-transformation of $\mathrm{Lp}(\mathrm{a})$ data; $\mathrm{B}=-0.542, p=0.036$ ). Similar results were obtained using the mean KIV repeat number in the case two apo(a) isoforms were expressed (rho $=-0.369, p=0.021$ and rho $=0.054, p=0.74$, respectively).

\subsection{Association with common LPA SNPS}

In the combined initiation and control group, carriers of the minor rs10455872 allele had a higher $\mathrm{Lp}(\mathrm{a})$ level at baseline than non-carriers (172.2 (IQR 83.0-215.1) vs. 18.8 (IQR 8.5-78.8) $\mathrm{mg} / \mathrm{dL}$; $\mathrm{MWU}=100.0 ; \mathrm{Z}=4.151 ; p<0.001)$. In addition, 11 out of 12 carriers of the rs10455872 minor allele had the LMW phenotype $(p<0.001$ by 
Pearson Chi-Square). There was a strong correlation of this SNP with the LMW $v s$. HMW phenotype $(\mathrm{r}=-0.515, p<0.001)$ as well as with the KIV repeat number of the smallest apo(a) isoform $(r=-0.523$, $p<0.001$ ). Lp(a) levels in carriers of the minor rs41272110 allele were similar to those in the non-carriers (23.6 (IQR 12.5-90.6) vs. 29.0 (IQR 8.6-114.3) $\mathrm{mg} / \mathrm{dL} ; \mathrm{MWU}=654.5 ; \mathrm{Z}=0.488 ; p=0.63)$. There were no carriers of the minor rs 3798220 allele in the initiation group.

We assessed the association of change in Lp(a) levels with the LPA SNPs rs10455872 and rs41272110. The changes in Lp(a) levels over time were not significantly different between the rs10455872 carriers $v$ s. non-carriers in the initiation group (MWU $=29.0 ; \mathrm{Z}=-1.855$; $p=0.066$ ) nor in the control group (MWU $=125.5 ; \mathrm{Z}=-0.336$; $p=0.74$ ). For rs 41272110 there were also no significant differences in Lp(a) changes over time between carriers vs. non-carriers $(\mathrm{MWU}=164.5 ; \quad \mathrm{Z}=-0.239 ; \quad p=0.81 \quad$ and $\quad \mathrm{MWU}=134.5$; $\mathrm{Z}=-1.030 ; p=0.31$, respectively).

\subsection{Association with LDL-cholesterol}

In the initiation group, LDL-C levels decreased from 5.3 (IQR 4.4-6.4) to 3.1 (IQR 2.6-4.1) $\mathrm{mmol} / \mathrm{L}(\mathrm{Z}=5.373 ; p<0.001$ ) on statin therapy. In the control group, LDL-C remained stable between the two time points (2.6 (IQR 2.2-2.9) $\mathrm{mmol} / \mathrm{L}$ vs 2.7 (IQR 2.2-3.1) $\mathrm{mmol} / \mathrm{L}$ $(\mathrm{Z}=1.277 ; p=0.202)$ ). Qualitatively similar results were found for 'true LDL-C' levels, which were derived from LDL-C by correcting for Lp (a)-cholesterol. We found no association of LDL-C or 'true LDL-C' and Lp (a) at baseline in the initiation group (rho $=-0.109 ; p=0.52$ and rho $=-0.293 ; p=0.074$, respectively). In the control group, LDL-C was not associated with $\mathrm{Lp}(\mathrm{a})$ levels at baseline (rho $=-0.014$; $p=0.93$ ) but 'true LDL-C' did (rho $=-0.600 ; p<0.001$ ). In both groups, there was no association between change in $\mathrm{Lp}(\mathrm{a})$ and change in LDL-C in both groups (rho $=-0.030 ; p=0.86$ and rho $=-0.015$; $p=0.93$, respectively), nor with 'true LDL-C' (rho $=-0.136 ; p=0.39$ and rho $=-0.092 ; p=0.58$, respectively).

\section{Discussion}

Our data suggest that initiation of statin treatment increases Lp(a) levels in patients with dyslipidemia, but exclusively in patients with the LMW apo(a) phenotype. Initiation of statin treatment increased Lp(a) levels by $11.1 \%$ in the entire group, which is in line with results of two recent meta-analyses, showing an increase of $10.6 \%$ [29] and $4.14 \mathrm{mg} / \mathrm{dL}$ [30]. This average increase in our patients was mainly driven by the presence of an LMW apo(a) phenotype in whom the increase of Lp(a) level was roughly almost $50 \%$. We did not find an interaction between Lp(a) change and common LPA SNPs across the groups, which may have been due to the low number of minor allele carriers in our study population. A recent meta-analysis of seven randomized controlled statin trials with cardiovascular disease outcomes showed no overall effect of 1-year statintreatment on plasma Lp(a) levels [31], as Lp(a) levels significantly increased in 3 trials but decreased in 3 other trials. Compared to those trials, our study included predominantly familial hypercholesterolemia patients with relatively high baseline Lp(a) levels, who were statin treated for shorter time. Moreover, we were able to correlate changes in Lp(a) levels with the number of apo(a) KIV repeats, not only when used as a categorical but also as a continuous variable in the analysis. Lp(a) levels increased to a lesser extent with higher KIV repeat number, which was independent of baseline levels. To our knowledge, we are the first to show that the effect of statin treatment on plasma Lp(a) levels in individual patients depends on the apo(a) phenotype, with Lp(a) levels increasing exclusively in carriers of at least one apo(a) isoform with $\leq 22 \mathrm{KIV}$ repeats.

The mechanism of this statin-induced increase in $L p(a)$ level remains unclear. The diagnosis of dyslipidemia and initiation of a statin could hypothetically have led to increased risk awareness, resulting in a change in lifestyle including for example less saturated fat-intake. Reduced fat intake and a shift from dietary saturated to unsaturated fat have been previously associated with increased Lp(a) levels [17]. Lp(a) levels increased more upon shift to dietary unsaturated fat in subjects with high baseline Lp(a) [18], and hence presumably in subjects with LMW apo(a) phenotype than with HMW apo(a) phenotype. Alternatively, statins may indirectly increase plasma Lp(a) levels by upregulation of apo(a) synthesis or reducing $\mathrm{Lp}(\mathrm{a})$ clearance. Since smaller apo(a) isoforms with lower number of KIV repeats are secreted more efficiently and are cleared less efficiently than larger apo(a) isoforms [32-35], the statin effect may be more pronounced in subjects with LMW than the HMW apo(a) phenotype.

We found that 'true LDL-C' negatively associated with Lp(a) levels in patients from the control group, which are stably on statin treatment, but this did not reach statistical significance for patients in the initiation group at baseline, when not on cholesterol-lowering medication yet. The higher the levels of apo(a), the more apo(a) may become bound to LDL particles, thus explaining the negative association between 'true LDL-C' and Lp(a). That this reached statistically significant levels in the control group only may indicate some degree of overcorrection for Lp(a)-cholesterol, which will have a stronger effect after than before LDL-C is lowered by statin treatment.

It has long been known that statin treatment affects Lp(a) levels not in parallel with the lowering of LDL-C. Here, we show that statins even increase Lp(a) levels while lowering LDL. In addition, we found LDL-C and 'true LDL-C' levels not to be associated with Lp(a) levels over time after starting statin treatment. In contrast, monoclonal antibodies against proprotein convertase subtilisin kexin type 9 (PCSK9) have been shown to reduce Lp(a) levels in parallel with the lowering of LDL-C and apoB100 [36,37].Recently, Watts et al. [38] showed that PCSK9 inhibitors lower Lp(a) levels in healthy individuals by reducing the production of Lp(a)-associated apo(a), whereas it increases the clearance of Lp(a) when LDL-receptor expression is further upregulated by statin treatment, The mechanism underlying the upregulation of Lp(a) levels upon treatment of FH patients with a statin remains to be established.

\subsection{Strenghts and limitations of the study}

Limitations of this study are the small number of subjects included, the various types and dosages of statins and duration of treatment, the lack of data on lifestyle (in particular diet), and the observational nature of the study lacking randomization. Although we found no significant difference in baseline $\mathrm{Lp}(\mathrm{a})$ levels between the initiation and the control group, the Lp(a) level in the LMW group was higher in the control vs initiation group. Patients with high Lp(a) levels may be overrepresented in our control group of dyslipidemia patients who are frequently invited back at the outpatient clinic of our tertiary center despite being on stable statin treatment. However, this did not affect the main outcome. Strengths of this study include Lp(a) measurements of all samples in a single run and without repeated freezing-thawing cycles, the inclusion of a control group with constant statin treatment and the determination of the apo(a) phenotypes and common Lp(a) SNPs.

\subsection{Conclusions}

Our data shows that statin treatment increases Lp(a) levels in dyslipidemic patients with the LMW apo(a) phenotype. This increase was not associated with common LPA SNPs or change in LDL cholesterol. To understand the effects of statins and other drugs on $\mathrm{Lp}$ (a) levels, more research is needed on $\mathrm{Lp}(\mathrm{a})$ metabolism, including synthesis, clearance and turnover of apo(a).

\section{Conflicts of interest}

JRVL reports that the institution received honorary fees from Aegerion Pharmaceuticals Inc., United States and grants from Sanofi, France, and the Dutch Heart Foundation (Netherlands), all outside the submitted work. All other authors have nothing to disclose. 


\section{Financial support}

This study was supported in part by a grant from the Erasmus Medical Center Efficiency Research funding program (NL 42635.078.13).

\section{References}

[1] F. Kronenberg, G. Utermann, Lipoprotein(a): resurrected by genetics, J. Intern. Med. 273 (1) (2013) 6-30.

[2] D. Saleheen, et al., Apolipoprotein(a) isoform size, lipoprotein(a) concentration, and coronary artery disease: a mendelian randomisation analysis, Lancet Diabetes Endocrinol 5 (7) (2017) 524-533.

[3] P. Deloukas, et al., Large-scale association analysis identifies new risk loci for coronary artery disease, Nat. Genet. 45 (1) (2013) 25-33.

[4] P.R. Kamstrup, et al., Genetically elevated lipoprotein(a) and increased risk of myocardial infarction, Jama 301 (22) (2009) 2331-2339.

[5] R. Alonso, et al., Lipoprotein(a) levels in familial hypercholesterolemia: an important predictor of cardiovascular disease independent of the type of LDL receptor mutation, J. Am. Coll. Cardiol. 63 (19) (2014) 1982-1989.

[6] A. Cai, et al., Lipoprotein(a): a promising marker for residual cardiovascular risk assessment, Dis. Markers 35 (5) (2013) 551-559.

[7] G. Utermann, et al., Lp(a) glycoprotein phenotypes. Inheritance and relation to Lp (a)-lipoprotein concentrations in plasma, J. Clin. Investig. 80 (2) (1987) 458-465.

[8] A.M. Scanu, G.M. Fless, Lipoprotein (a). Heterogeneity and biological relevance, J. Clin. Investig. 85 (6) (1990) 1709-1715.

[9] E. Boerwinkle, et al., Apolipoprotein(a) gene accounts for greater than $90 \%$ of the variation in plasma lipoprotein(a) concentrations, J. Clin. Investig. 90 (1) (1992) $52-60$.

[10] C. Lackner, J.C. Cohen, H.H. Hobbs, Molecular definition of the extreme size polymorphism in apolipoprotein(a), Hum. Mol. Genet. 2 (7) (1993) 933-940.

[11] H.G. Kraft, et al., The apolipoprotein (a) gene: a transcribed hypervariable locus controlling plasma lipoprotein (a) concentration, Hum. Genet. 90 (3) (1992) 220-230.

[12] D.L. Rainwater, et al., Characterization of the genetic elements controlling lipoprotein(a) concentrations in Mexican Americans. Evidence for at least three controlling elements linked to LPA, the locus encoding apolipoprotein(a), Atherosclerosis 128 (2) (1997) 223-233.

[13] F. Kronenberg, Human genetics and the causal role of lipoprotein(a) for various diseases, Cardiovasc. Drugs Ther. 30 (1) (2016) 87-100.

[14] W. Lu, et al., Evidence for several independent genetic variants affecting lipoprotein (a) cholesterol levels, Hum. Mol. Genet. 24 (8) (2015) 2390-2400.

[15] J.P. Chretien, et al., Three single-nucleotide polymorphisms in LPA account for most of the increase in lipoprotein(a) level elevation in African Americans compared with European Americans, J. Med. Genet. 43 (12) (2006) 917-923.

[16] A. Brandstatter, et al., Decrease of Lp(a) during weight reduction in obese children is modified by the apo(a) kringle-IV copy number variation, Int. J. Obes. 33 (10) (2009) 1136-1142.

[17] B.A. Clevidence, et al., Plasma lipoprotein (a) levels in men and women consuming diets enriched in saturated, cis-, or trans-monounsaturated fatty acids, Arterioscler. Thromb. Vasc. Biol. 17 (9) (1997) 1657-1661.

[18] N. Faghihnia, et al., Changes in lipoprotein(a), oxidized phospholipids, and LDL subclasses with a low-fat high-carbohydrate diet, J. Lipid Res. 51 (11) (2010) 3324-3330.

[19] F. Kronenberg, et al., Lipoprotein(a) serum concentrations and apolipoprotein(a) phenotypes in mild and moderate renal failure, J. Am. Soc. Nephrol. 11 (1) (2000)
$105-115$

[20] S. van Wissen, et al., Long term statin treatment reduces lipoprotein(a) concentrations in heterozygous familial hypercholesterolaemia, Heart 89 (8) (2003) 893-896.

[21] S. Tsimikas, et al., High-dose atorvastatin reduces total plasma levels of oxidized phospholipids and immune complexes present on apolipoprotein B-100 in patients with acute coronary syndromes in the MIRACL trial, Circulation 110 (11) (2004) 1406-1412.

[22] A.V. Khera, et al., Lipoprotein(a) concentrations, rosuvastatin therapy, and residual vascular risk: an analysis from the JUPITER Trial (Justification for the Use of Statins in Prevention: an Intervention Trial Evaluating Rosuvastatin), Circulation 129 (6) (2014) 635-642.

[23] S. Van Wissen, et al., Long term statin treatment reduces lipoprotein(a) concentrations in heterozygous familial hypercholesterolaemia, Heart 89 (8) (2003) 893-896.

[24] A. Matsunaga, et al., Effect of simvastatin on receptor mediated metabolism of low density lipoprotein in Guinea pigs, Atherosclerosis 90 (1) (1991) 31-37.

[25] N.J. Viney, et al., Relationship between "LDL-C", estimated true LDL-C, apolipoprotein B-100, and PCSK9 levels following lipoprotein(a) lowering with an antisense oligonucleotide, J Clin Lipidol 12 (3) (2018) 702-710.

[26] R. Vongpromek, et al., Lipoprotein(a) levels are associated with aortic valve calcification in asymptomatic patients with familial hypercholesterolaemia, J. Intern. Med. 278 (2) (2015) 166-173.

[27] K.A. Berk, et al., Effect of diet-induced weight loss on lipoprotein(a) levels in obese individuals with and without type 2 diabetes, Diabetologia 60 (6) (2017) 989-997.

[28] J. Prins, et al., The identification and significance of a Thr- $>$ Pro polymorphism in kringle IV type 8 of apolipoprotein(a), Thromb. Haemostasis 77 (5) (1997) 949-954.

[29] C. Yeang, et al., Effect of therapeutic interventions on oxidized phospholipids on apolipoprotein B100 and lipoprotein(a), J Clin Lipidol 10 (3) (2016) 594-603.

[30] A. Sahebkar, et al., Comparison of the effects of fibrates versus statins on plasma lipoprotein(a) concentrations: a systematic review and meta-analysis of head-tohead randomized controlled trials, BMC Med. 15 (1) (2017) 22.

[31] P. Willeit, et al., Baseline and on-statin treatment lipoprotein(a) levels for prediction of cardiovascular events: individual patient-data meta-analysis of statin outcome trials, Lancet 392 (10155) (2018) 1311-1320.

[32] J. Wang, et al., Determinants of human apolipoprotein [a] secretion from mouse hepatocyte cultures, J. Lipid Res. 42 (1) (2001) 60-69.

[33] E.M. Lobentanz, et al., Intracellular metabolism of human apolipoprotein(a) in stably transfected Hep G2 cells, Biochemistry 37 (16) (1998) 5417-5425.

[34] C. Brunner, et al., The number of identical kringle IV repeats in apolipoprotein(a) affects its processing and secretion by HepG2 cells, J. Biol. Chem. 271 (50) (1996) 32403-32410.

[35] D.C. Chan, et al., Lipoprotein(a) particle Production as a Determinant of plasma lipoprotein(a) concentration across varying apolipoprotein(a) isoform Sizes and background cholesterol-lowering therapy, J Am Heart Assoc 8 (7) (2019) p. e011781.

[36] F.J. Raal, et al., Inhibition of PCSK9 with evolocumab in homozygous familial hypercholesterolaemia (TESLA Part B): a randomised, double-blind, placebo-controlled trial, Lancet 385 (9965) (2015) 341-350.

[37] G.M. Kostner, et al., HMG CoA reductase inhibitors lower LDL cholesterol without reducing Lp(a) levels, Circulation 80 (5) (1989) 1313-1319.

[38] G.F. Watts, et al., Controlled study of the effect of proprotein convertase subtilisin kexin type 9 inhibition with evolocumab on lipoprotein(a) particle kinetics, Eur. Heart J. 39 (27) (2018) 2577-2585.

[39] J. Besseling, B. Sjouke, J.J. Kastelein, Screening and treatment of familial hypercholesterolemia - lessons from the past and opportunities for the future (based on the Anitschkow Lecture 2014), Atherosclerosis 241 (2) (2015) 597-606. 